\title{
Alloxylon (Proteaceae), a new genus from New Guinea and eastern Australia
}

\author{
Peter H. Weston and Michael D. Crisp
}

\begin{abstract}
Weston, Peter H. ${ }^{1}$, and Crisp, Michael D. ${ }^{2}\left({ }^{1}\right.$ National Herbarium of New South Wales, Royal Botanic Gardens, Sydney NSW Australia 2000; ${ }^{2}$ Australian National Botanic Gardens, GPO Box 1777, Canberra ACT Australia 2601; present address: Division of Botany and Zoology. Australian National University, GPO Box 4, Canberra ACT 2601) 1991. Alloxylon (Proteaceae), a new genus from New Guinea and eastern Australia. Telopea 4(3): 497-507. Oreocallis sens. lat. consists of two distinct clades, one in South America, the other in Australasia, that together are likely to be paraphyletic. Newly sampled characters strongly support the monophyly of the Australasian group. We describe the new genus Alloxylon to accommodate the Australasian species of Oreocallis sens. lat. and revise its species. Alloxylon flammeum is described as new and new combinations are made for A. brachycarpum, A. wickhamii and A. pinnatum.
\end{abstract}

\section{Introduction}

Cladistic analyses of the subtribe Embothriinae of the family Proteaceae (Weston \& Crisp 1987, in prep.) show the genus Oreocallis $\mathrm{R}$. Br. sens. lat. to comprise two branches of an unresolved trichotomy. The third branch is the genus Telopea, a well corroborated clade of five species. The sister group to this trichotomy is Embothrium, the only other genus in the Embothriinae. According to these results, Oreocallis is likely to be paraphyletic, necessitating a nomenclatural rearrangement in order to ensure that all formal names apply to monophyletic groups. Two obvious nomenclatural alternatives are to (a) sink Telopea and Oreocallis into a single genus, or (b) elevate the two distinct groups within Oreocallis sens. lat. to generic rank. We have chosen the latter alternative for the following reasons. Firstly, Telopea and both clades in Oreocallis sens. lat. are more thoroughly characterised by synapomorphies than the group that includes all three of these clades. Option (b) is thus more likely to be nomenclaturally stable than option (a). Secondly, option (b) requires fewer nomenclatural changes than does option (a): only four species are moved into a new genus whereas option (a) requires either that five species of Telopea be moved to Oreocallis or that six species of Oreocallis be moved to Telopea (Telopea and Oreocallis were both established by Brown (1810) and so are equal in priority). Therefore we here establish the new genus Alloxylon to accomodate the three Australian and one New Guinean species formerly included in Oreocallis. Oreocallis sens. strict. comprises two species that broadly overlap in distribution in Peru and Ecuador, O. grandiflora and O. mucronata.

Since the publication of our first cladistic analysis of the Embothriinae (Weston \& Crisp 1987), knowledge of the wood, fruits, leaf anatomy and seedlings in the Embothriinae has improved considerably, providing a number of new characters for cladistic analysis. Moreover, we now realise that there are three species, one previously unnamed, in north-eastern Australia and New Guinea, not just one as we had previously believed, nor two as Sleumer (1954) had thought.

The data presented here were compiled from herbarium specimens, cultivated plants, field observations and literature. Specimens held by the following herbaria were 
examined: BRI, CANB, CBG, MEL, MO, NSW, QRS, SYD. All Australian species are in cultivation at the Royal Botanic Gardens Sydney.

Terms denoting form are taken from Stearn (1973), particularly his figure 19. Inflorescence terminology follows that of Johnson \& Briggs (1975) and Weston \& Crisp (1987), while leaf venation is described using the terms of Hickey (1973). Flower orientation with respect to the unit inflorescence axis is described as diagonal (opposed to anteroposterior) following Johnson \& Briggs (1963, 1975) and Weston \& Crisp (1987).

\section{Taxonomy}

\section{Alloxylon P. Weston $\mathcal{E}$ Crisp, gen. nov.}

Arbores parvae ad elatae, in silvis humidis clausis crescentes; cotyledonibus succulentis; bracteis involucralibus nullis; floribus zygomorphis, in alabastro leviter incurvis, roseis ad rubris; perianthio in alabastro plus minusve cylindrico, tubo proximali lineari et limbo distali introrso-obliquo subgloboso, tepalis cohaerentibus postice in tubo fisso post anthesin; polline triporato; pollinophoro valde introrso-obliquo, plus minusve late elliptico ad basin, late conico lateribus concavis, stigmate parvo circulari terminato; fructu folliculi, mesocarpio interiore lignoso, non late dehiscenti, post dehiscentiam cymbiformi; seminibus 6-14, ala seminis basalis longitudine latitudineque loculum fructus fere aequanti sed in seminibus distalibus successive breviore.

TYPE: Alloxylon flammeum P. Weston \& Crisp.

Cotyledons fleshy, epigeal, shortly auriculate, green after germination. First two seedling leaves opposite; all later leaves alternate, with spiral phyllotaxis. Small to tall rainforest trees. Trichomes unbranched. Glandular hairs absent. Adult leaves simple and entire or pinnate with entire leaflets, tapered to petiole. Intermediate leaves simple and entire or deeply pinnatisect or pinnate. Seedling leaves simple, deeply lobed or entire. Conflorescences axillary or terminal, pseudoracemose, laterally bearing flower-pairs (unit inflorescences sensu Johnson \& Briggs 1975), basitonic or acrotonic, solitary or aggregated into superconflorescences; involucral bracts absent; unit inflorescences lacking floral bracts and peduncles. Flowers zygomorphic, diagonally oriented with respect to unit inflorescence axis, gently incurved in bud, hermaphrodite, ornithophilous, pink to red; torus strongly oblique, lowest on posterior side of flower; perianth \pm cylindrical in bud, consisting of proximal linear claw and distal inward-oblique, subglobose limb; segments cohering posteriorly in split tube after anthesis; staminal filaments adnate to perianth segments; anthers circular, introrse, with connective projecting slightly beyond tips of loculi; pollen triporate; hypogynous glands connate, forming posterior, lunate nectary; gynoecium glabrous, $c$. as long as perianth; gynophore well-developed, half as long as style or more; ovary slightly swollen, containing numerous, laterally attached, winged, anatropous ovules; pollenpresenter strongly inward-oblique, \pm broad-elliptical at base, broadly conical with concave sides, terminated by small circular stigma. Fruit a follicle, narrow-ellipsoid to narrow-obovoid, with woody inner mesocarp, not opening widely, canoe-like on dehiscence. Seeds 6-14, interleaved by papery, dark brown interseminal layer; embryo flattened, fleshy; wing distal to embryo, narrow-oblong, truncate to rounded or acute; wing of basal seed nearly as long and wide as loculus of fruit but progressively shorter in distal seeds; raphe entering seed on outside edge of embryo, running along edge onto wing then running diagonally across wing to inside edge near distal tip, sharply reversing and running along inside margin of wing to embryo. 
Three species endemic in eastern Australia, and one endemic in New Guinea and the Aru Islands. This genus was formerly included in Oreocallis, which, in our delimitation, comprises two species in Peru and Ecuador. All species, but A. flammeum in particular, are showy flowering trees suitable for cultivation in mildly temperate to tropical areas. The generic name is derived from the Greek allo (other) and xylon (wood), referring to the wood anatomy of the genus, which is markedly different from that of other Proteaceae (Chattaway 1948; Papassotiriou, Weston \& Wilkins unpublished data).

\section{Key to the species}

1 Adult leaves mostly pinnate, some simple; leaf(let) apices attenuate; venation of terminal leaflets and simple leaves eucamptodromous; conflorescences 50-140flowered, solitary; pollen deep crimson

1. A. pinnatum

$1^{*}$ Adult leaves simple and entire; leaf apices obtuse or acute; venation brochidodromous; conflorescences 4-52-flowered, often grouped in superconflorescences; pollen yellow.

2 Flowers glabrous, dull pinkish red; conflorescences 4- 20-flowered, symmetrical to slightly asymmetrical; leaves coriaceous

2. A. wickhamii

2* Flowers covered in (sometimes very short) ferruginous hairs, pinkish red to bright orange-red; conflorescences (2-)10-52-flowered, strongly asymmetrical, the pedicels strongly curved so that all flowers are similarly oriented on the same side of conflorescence axis; leaves chartaceous.

3 Hairs mostly spreading, straight to curly, 0.1-0.5 mm long; young branchlets densely hairy

3. A. flammeum

3* Hairs appressed, straight, to $0.1 \mathrm{~mm}$ long; young branchlets sparsely to moderately hairy

4. A. brachycarpum

1. Alloxylon pinnatum (Maiden $\&$ Betche) P. Weston $\mathcal{E}$ Crisp, comb. nov.

BAsIONYM: Embothrium wickhamii W. Hill \& F. Muell. var. pinnatum Maiden \& Betche, Proc. Linn. Soc. New South Wales 35: 795 (1910).

TYPE CitATION: 'Dorrigo (J.L. Boorman; December, 1909).' LectotyPe (here designated): New South Wales: Dorrigo, J. L. Boorman s.n., Dec 1909 (NSW 235580); isolecto B, BRI, MEL (2 sheets), NSW (2 sheets).

SYNONYMS: Embothrium pinnatum (Maiden \& Betche) C. White, Proc. Roy. Soc. Queensland 60: 43 (1950).

Oreocallis pinnata (Maiden \& Betche) Sleumer, Bot. Jahrb. Syst. 76: 203 (1954).

Illustration: M. Flockton in Maiden, Forest Fl. N.S.W. 5: t. 167 (1911), as Embothrium wickhamii var. pinnatum.

Seedlings and juveniles differing from adults as follows: first 6-8 leaves simple, entire, ovate to elliptical (or rarely one of them 2-lobed), discolorous, (1.5-)3.5-5.0 cm long, (4-)10-25 mm wide, tapered to base, not petiolate, acute to acuminate; venatation brochidodromous, with secondary veins diverging at 30-70 degrees from midvein, secondary and some lower order veins protruding slightly when dried; later 
seedling leaves simple and entire to 12 th-22nd node, narrow-elliptical to -obovate, discolorous, $7.5-18 \mathrm{~cm}$ long, 13-55 mm wide, with attenuate base, petiolate, acuminate; venation brochidodromous, with secondary veins diverging at 25-40 degrees from midvein; intermediate leaves pinnate with 2-9 leaflets, discolorous, (10-)15-35 $\mathrm{cm}$ long; venation of terminal leaflet brochidodromous.

Adults: trees to $24 \mathrm{~m}$ high and $0.6 \mathrm{~m}$ d.b.h., not buttressed. Bark grey-brown, with a dense cover of fine lenticels. Hairs short, mostly $0.1-0.45 \mathrm{~mm}$ long, ferruginous when young but aging to grey, antrorse, straight. Young branchlets sparsely to moderately hairy, soon glabrescent. Leaves mostly pinnate with few simple entire leaves (particularly just below conflorescences), concolorous to slightly discolorous, chartaceous, bright green when immature; petiole sparsely to moderately hairy when immature (densest on adaxial surface and at base), glabrescent to sparsely hairy when mature; laminae narrow-elliptical to narrow-ovate, attenuate at base and tip, with flat to slightly recurved margins, sparsely hairy when immature, glabrescent when mature; midvein protruding prominently and \pm equally on both surfaces; tertiary and lower order veins sunken; pinnate leaves with 2-11 leaflets, $10-50 \mathrm{~cm}$ long; petiole $35-145$ $\mathrm{mm}$ long; lateral leaflets usually asymmetrical at base, often slightly falcate, 5.0-14.5 $\mathrm{cm}$ long, 8-21 mm wide, forming angle of 22-30 degrees at base; venation usually brochidodromous or sometimes eucamptodromous, with secondary veins diverging at 30-45 degrees; secondary veins usually sunken; terminal leaflet with lower length:width ratio than lateral leaflets, occasionally asymmetrical or deeply 2-3-lobed, more attenuate at base than lateral leaflets, 8-18 cm long, 17-34 mm wide, forming angle of 35-45 degrees at base; venation usually eucamptodromous or occasionally brochidodromous, with secondary veins diverging at 13-30 $(-40)$ degrees; secondary veins protruding proximally but sunken distally, most prominent on adaxial surface; simple leaves similar to terminal leaflets but with higher length:width ratio, $8-23 \mathrm{~cm}$ long, 11-31 mm wide, forming angle of 22-30 degrees at base; petiole 8-45 mm long. Conflorescences 50-140-flowered, terminal or rarely axillary, acrotonic with 1-11 basal leaves (uppermost $0-11$ being simple) or rarely basitonic, solitary, symmetrical or slightly asymmetrical; axis moderately hairy; bracts of flower-pairs mostly lineartriangular to -oblong, caducous or persistent, but often basal $1-6(-20)$ flower-pairs subtended by reduced simple entire leaves. Flowers bright pink, glabrous; pedicels 30$60 \mathrm{~mm}$ long; perianth 30-38 mm long, widest below middle and tapering slightly to ends of claw; pollen deep crimson; style c. twice as long as gynophore. Body of follicle 5-9 cm long; seeds 8-11. Figure 1.

FLOWERING PERIOD: November to January.

DistribUTION AND HABITAT: Australia; vicariously distributed in the Dorrigo area, New South Wales, and the McPherson Range, New South Wales and Queensland (Figure 2a). Mountain slopes and plateaux, 700-1250 m altitude, on soils derived from sedimentary rocks, metasediments, and volcanics, in warm-temperate rainforest dominated for example by Ceratopetalum apetalum, rarely in wet eucalypt forest.

CONSERVATION STATUS: 3RC. Both parts of the distribution of $A$. pinnatum cover comparatively small geographic ranges. Moreover, it is not a common species where it does occur. Surprisingly, it is not known from any of the warm-temperate rainforests that occur between the disjunct parts of its distribution. Some of these, such as the Washpool rainforests, are quite extensive.

SELECTED SPECIMENS (40 examined): QuEENSLAND: Moreton: Tomewin, near Springbrook, $28^{\circ} 14^{\prime}$ S, $153^{\circ} 23^{\prime} \mathrm{E}$, Andersen s.n., 8 Nov 1968 (BRI); Roberts Plateau, Shirley 645/18, Feb 1918 (BRI, NSW). New South Wales: North Coast: Tyalgum Ridge, Limpinwood Nature Reserve, $28^{\circ} 18^{\prime} \mathrm{S}$, 15308'30"E, Coveny 9935 \& Haegi, 3 Dec 1977 (BRI, NSW); Woolgoolga Creek dam, Floyd 1957, 15 May 1983 (NSW); Dorrigo National Park, $8 \mathrm{~km}$ along road to Never Never from ranger 


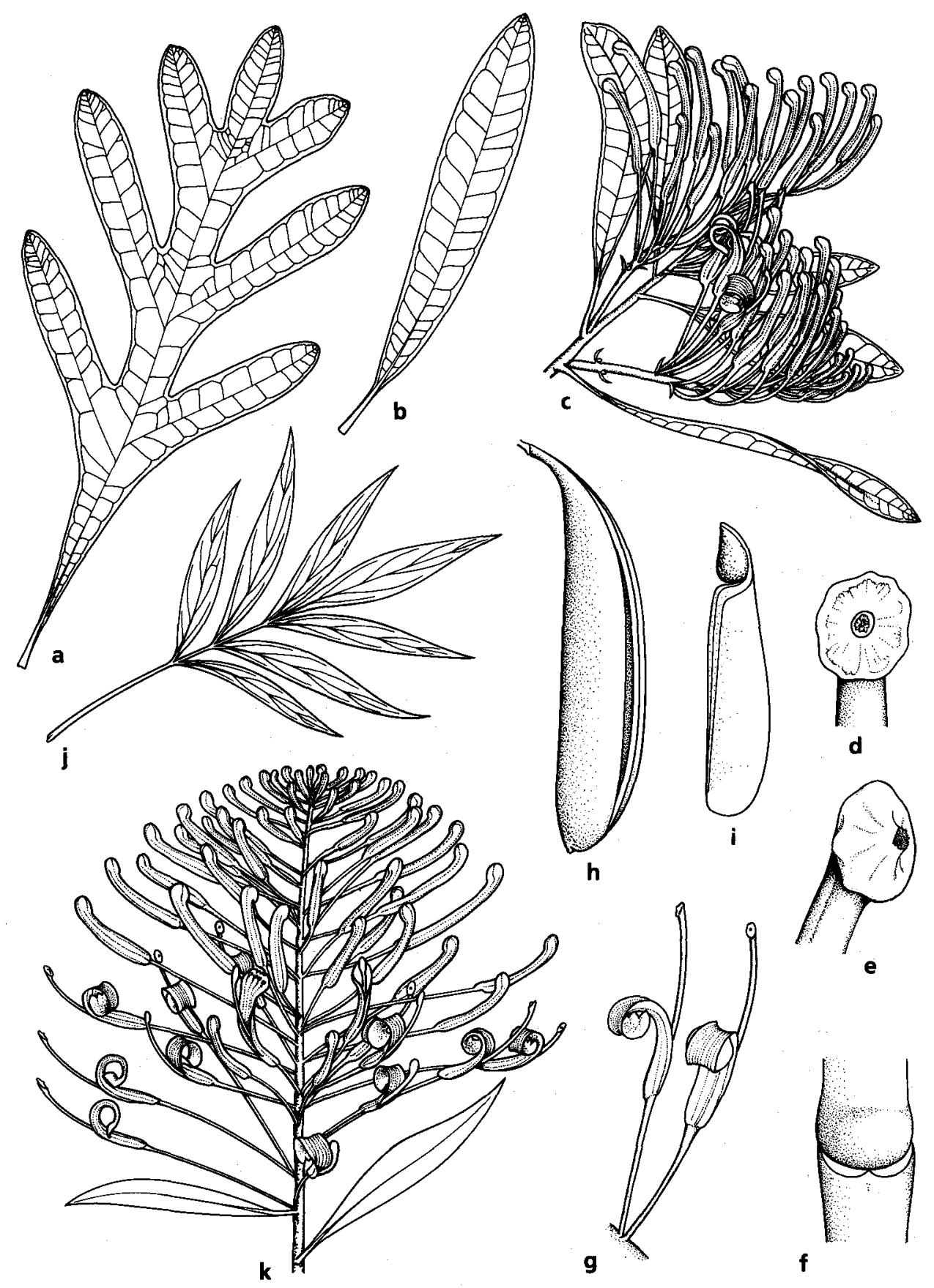

Figure 1. a, juvenile leaf of A. flammeum (x $1 / 4)$; b, adult leaf of A. flammeum (x $1 / 4)$; c, superconflorescence of $A$. flammeum (x $1 / 2)$; d-e, pollen presenter of $A$. flammeum (x 5); $\mathbf{f}$, hypogynous gland of $A$. flammeum (x 5); $\mathbf{g}$, flower pair of A. flammeum (x $1 / 2)$; h, fruit of $A$. wickhamii (x $1 / 2)$; $\mathbf{i}$, seed of $A$. wickhamii (x $1 / 2)$; $\mathbf{j}$, pinnate leaf of $A$. pinnatum $\left(x^{1 / 4}\right) ; \mathbf{k}$, conflorescence of $A$. pinnatum. (Drawn from Constable, cultivated, Royal Botanic Gardens Sydney, 11.1958 (a-b), Weston 402 (c), Weston $406(\mathrm{~d}-\mathrm{g})$, Smith 4162 A (h-i), Floyd 1957 (j), Salasoo $1878(\mathrm{k})$.) 
station, Crisp 7590 \& Taylor, 3 Nov 1984 (CBG, MEL, NSW). Northern Tablelands: Mt Hyland Nature Reserve, $30^{\circ} 10^{\prime} \mathrm{S}, 152^{\circ} 28^{\prime} \mathrm{E}$, Richards 93 \& Weston, 1 Feb 1988 (BRI, CBG, K, MEL, MO, NSW, QRS). Cultivated: Epping (New South Wales), Hazlewood s.n., 2 Dec 1958 (NSW 48284).

\section{Alloxylon wickhamii (W. Hill \& F. Muell.) P. Weston \& Crisp, comb. nov.}

BASIONYM: Embothrium wickhamii W. Hill \& F. Muell., Fragm. 8: 164 (1874) (as E. wickhami).

TYPE CITATION: 'In monte Bellenden-Kerii, altitudine circiter 2500 pedum, ubi Heliciae Youngianae adsociatum; Walt. Hill.'

LECTOTYPE (here designated): QuEENSLAND: Bellenden Ker, $2500 \mathrm{ft}$ altitude, W. Hill 204, - (MEL 56276).

Residual syntyPE: Bellenden Ker, Qld, W. Hill 41, - (MEL 56277).

SYNONMM: Oreocallis wickhamii (W. Hill \& F. Muell.) Sleumer, Bot. Jahrb. Syst. 76: 203 (1955).

Seedlings and juveniles differing from adults as follows: first pair of leaves narrowovate to -elliptical, acute, $6.5-8.0 \mathrm{~cm}$ long, $11-22 \mathrm{~mm}$ wide, chartaceous, not petiolate but tapered to base, bright green when immature; later seedling leaves progressively having higher length:breadth ratio, widest part closer to tip, less acute, longer, petiolate from c. 10th node; intermediate leaves narrow- to linear-obovate to -oblong, acute to obtuse, to $41 \mathrm{~cm}$ long, 21-60 mm wide, chartaceous, pinkish purple when immature, with undulate margins.

Adults: trees to $30 \mathrm{~m}$ high and $0.6 \mathrm{~m}$ d.b.h. Bark grey, reticulately and shallowly fissured with brown lenticels. Hairs minute, mostly $<0.1 \mathrm{~mm}$ long, ferruginous to grey, appressed, straight, virtually restricted to dormant buds. Branchlets glabrous to sparsely hairy when immature, soon glabrescent. Leaves simple, entire, 5-17 cm long, 11-34 mm wide, discolorous, coriaceous, pinkish purple when immature; petiole 12$30 \mathrm{~mm}$ long, glabrous to sparsely hairy when immature, glabrescent when mature; lamina mostly narrow-obovate or occasionally narrow-elliptical, forming angle of 3050 degrees at base, obtuse or rounded at apex, with flat margins, glabrous to sparsely hairy when immature, glabrescent when mature; venation brochidodromous, with secondary veins diverging at 55-70 degrees; midvein protruding slightly on adaxial surface and prominently on abaxial surface; lower order veins sunken. Conflorescences 4-20-flowered, terminal or axillary, basitonic or acrotonic with 1-10 basal leaves, solitary or aggregated in superconflorescences of 2-5 at ends of branches, symmetrical to slightly asymmetrical; axis sparsely hairy; bracts of flower-pairs mostly narrow-triangular to -oblong but often the basal 1-2 flower-pairs subtended by reduced leaves, persistent. Flowers dull pinkish red, glabrous; pedicels (15-)20-40 mm long; perianth 25-40 mm long, widest at base and distinctly tapering to tip of claw; pollen yellow; style c. twice as long as gynophore. Body of follicle 5.5-12 cm long; seeds 611. Figure 1.

FLOWERING PERIOD: October to November.

DisTRIBUTION AND HABITAT: Australia; north-eastern Queensland from Mt Bartle Frere to Cape Tribulation (Figure $2 \mathrm{~b}$ ). Mountainous areas, on soils derived from granite or metasediments, from 200 to $1220 \mathrm{~m}$ altitude, in tropical rainforest.

CONSERVATION STATUS: Not rare or endangered; it is fairly common through a relatively large area of the rainforests of the Cairns region.

The names Embothrium wickhamii and Oreocallis wickhamii were misapplied to Alloxylon flammeum until very recently. The two species were either treated as con- 
specific (e.g. Sleumer 1954, Weston \& Crisp 1987) or A. wickhamii was regarded as an undecribed species (e.g. Sankowsky et al. 1986).

SELECTED SPECIMENS (28 examined): QUEENSLAND: Cook: Gold Hill, summit ridge, $16^{\circ} 05^{\prime} \mathrm{S}, 145^{\circ} 17^{\prime} \mathrm{E}$, Weston 463, Sankowsky \& Hind, 12 Aug 1986 (NSW); Timber Reserve 55 Whyanbeel, 16 20'S, $145^{\circ} 20^{\prime} \mathrm{E}$, Hyland 7748,9 Oct 1974 (BRI, CANB, NSW, QRS); State Forest Reserve 607, Bridle Logging Area, $16^{\circ} 58^{\prime} \mathrm{S}, 145^{\circ} 37^{\prime} \mathrm{E}$, Gray 3745, 29 Nov 1984 (NSW, QRS); State Forest Reserve 194, Compartment $52,17^{\circ} 15^{\prime} \mathrm{S}, 145^{\circ} 25^{\prime} \mathrm{E}$, Hyland 3133, 22 Nov 1974 (BRI, CANB, NSW, QRS); WNW ridge of Mt Bartle Frere above Bobbin Bobbin Falls, $17^{\circ} 23^{\prime} \mathrm{S}, 145^{\circ} 46^{\prime} \mathrm{E}$, Weston 954, Sankowsky \& Hind, 23 Aug 1986 (NSW). Cultivated: Bushland Nursery, Sunnybank Hills (Queensland), Lebler s.n., 7 Dec 1973 (BRI).

\section{Alloxylon flammeum $P$. Weston $\mathcal{E}$ Crisp, sp. nov.}

Arbores ad $33 \mathrm{~m}$ alti et $0.6 \mathrm{~m}$ d.b.h.; pilis brevibus, plerumque $0.1-0.5 \mathrm{~mm}$ longis, ferrugineis juventute sed aetate provecta canis, plerumque patentibus, rectis ad crispis; ramulis juvenilibus pilis densis, demum glabrescentibus; plantulae foliis primo ad secundo vel quarto profunde trilobatis vel bilobatis, postea ad nodum $20-35$ simplicibus integrisque, angusto-ellipticis; foliis intermediis primo anguste ad profunde pinnatisectis lobis $2-9$, postea integris lineare ellipticis ad lineare obovatis; foliis adultis simplicibus integris, 8-25 cm longis, $20-25 \mathrm{~mm}$ latis, discoloribus chartaceis, immaturis prasinis; lamina angusto-elliptica ad angusto-obovata, ad basin angulum $22-55^{\circ}$

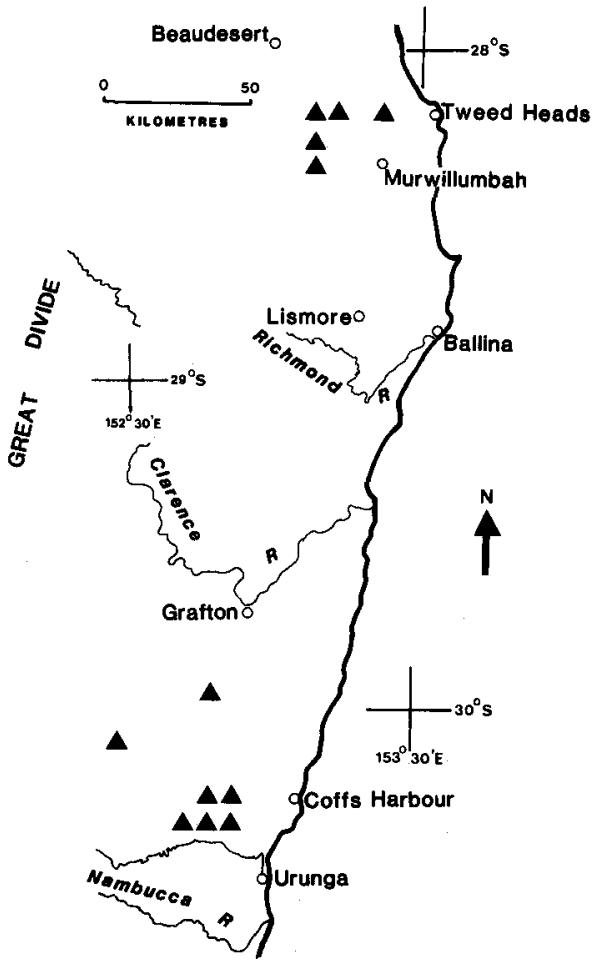

a

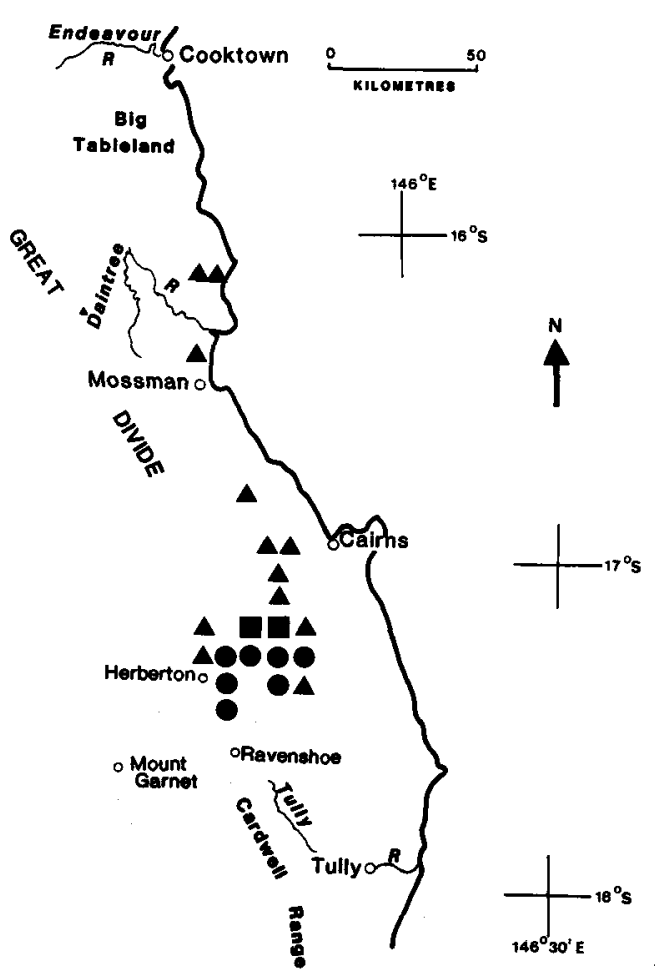

b

Figure 2. Distributions of species of Alloxylon; all symbols represent presence of one or more collection localities in a $5 \times 5$ minute grid square. a, A. pinnatum (south-eastern Queensland and northern New South Wales); $\mathbf{b}, A$. wickhamii (triangle), A. flammeum (circle), and both of these species (square) (northern Queensland). 
formanti, obtusa ad acuta; indumento costae aetate immatura pilis moderato vel denso, aetate matura glabrescenti; venatione brochidodroma, venis secundariis sub angulo circa $50-70^{\circ}$ patentibus; folii pagina abaxiali costa leviter protrudenti, venis depressis; conflorescentiis floribus 10-52, pro parte maxima axillaribus vel ramifloris, basitonicis vel rarissime acrotonicis foliis 1-2 basalibus, valde asymmetricis, 2-20 in superconflorescentias ad extremum ramorum plerumque aggregatis, rachide pilis densis; floribus vivide miniatis, pedicellis $25-43 \mathrm{~mm}$ longis, indumento moderate denso; perianthio $35-50 \mathrm{~mm}$ longo, infra medium latissimo et ungue versus extrema ambo leviter contracto, extus indumento moderate denso; polline flavo.

HoloTYPE: QuEensland: Cook: Tolga Scrub, $17^{\circ} 14^{\prime} \mathrm{S}, 145^{\circ} 29^{\prime} \mathrm{E}, \mathrm{G}$. G. Sankowsky $626 \&$ P. Radke, Aug 1987 (NSW 235579).

ISOTYPES: BRI, CBG, K, MEL.

[Embothrium wickhamii auct. non W. Hill \& F. Muell.: Bailey, Queensland Agric. J. 5: 403 (1899)]

Illustration: Bailey, Queensland Agric. J. 5: t.143 (1899), as Embothrium wickhamii.

Seedlings and juveniles differing from adults as follows: young stems sparsely to moderately hairy, soon glabrescent. First $2-4$ seedling leaves deeply trilobed or less frequently bilobed, $3.5-7.0 \mathrm{~cm}$ long, tapered to base, not petiolate, acute, sparsely hairy when immature, glabrescent when mature; later seedling leaves simple and entire to 20-35th node, narrow-elliptical, $6.5-18.0 \mathrm{~cm}$ long, $13-22 \mathrm{~mm}$ wide, petiolate from c. 10th node, acute; early intermediate leaves shallowly to deeply pinnatisect with 2-9 lobes, (18-)30-50 cm long; midveins of lobes protruding but less prominently so than primary midvein, mostly diverging at 30-40 degrees from primary midvein; later intermediate leaves entire, linear-elliptical to -obovate, $25-40 \mathrm{~cm}$ long, 33$45 \mathrm{~mm}$ wide.

Adults: trees to $33 \mathrm{~m}$ high and $0.6 \mathrm{~m}$ d.b.h. Bark grey, reticulately and shallowly fissured with prominent brown lenticels. Hairs short, mostly $0.1-0.5 \mathrm{~mm}$ long, ferruginous when young but aging to grey, mostly spreading, straight to curly. Young branchlets densely hairy, eventually glabrescent with age. Leaves simple, entire, 8-25 $\mathrm{cm}$ long, 20-45 mm wide, discolorous, chartaceous, bright green when immature; petiole 15-25 mm long, moderately to densely hairy when immature, sparsely so at base but otherwise glabrescent when mature; lamina narrow-elliptical to narrowobovate, forming angle of 22-55 degrees at base, obtuse to acute, with flat to slightly recurved margins, with moderately to densely hairy midvein but otherwise sparsely hairy when immature, glabrescent when mature; venation brochidodromous, with secondary veins diverging at 50-70 degrees; midvein protruding slightly on adaxial surface and prominently on abaxial surface; lower order veins sunken. Conflorescences 10-52-flowered, mostly axillary or ramiflorous, basitonic or very rarely acrotonic with 1-2 basal leaves, mostly aggregated in superconflorescences of 2-20 at ends of branches, strongly asymmetrical, pedicels curved so that all flowers are similarly oriented on same side of conflorescence axis; axis densely hairy; bracts of flower-pairs narrowtriangular to -oblong, mostly persistent. Flowers bright orange-red; pedicels $25-43 \mathrm{~mm}$ long, moderately hairy; perianth $35-50 \mathrm{~mm}$ long, widest below middle and tapering slightly to ends of claw, moderately hairy on outside; pollen yellow; style c. twice as long as gynophore. Body of follicle $7-10 \mathrm{~cm}$ long; seeds 8-10. Figure 1.

Chromosone number: $2 n=22$ (Johnson \& Briggs 1963: 24, cited as Oreocallis wickhamii; voucher Constable NSW 48283).

FLOWERING PERIOD: August to October. 
Distribution AND haBitat: Australia; restricted to the Atherton Tableland, north-eastern Queensland, from Danbulla south to the Upper Barron River (Figure 2b). Plateau summit at 700-820 $\mathrm{m}$ altitude, on basalt-derived soil, in tropical rainforest.

CONSERVATION STATUS: 2VC. The habitat of A. flammeum has been cleared almost completely for agriculture. It survives in the wild in a few remnant patches of rainforest near Atherton, including some small national parks. It is much sought after as a horticultural subject and cultivated plants probably outnumber those remaining in the wild.

Since last century, A. flammeum has been misidentified as $A$. wickhamii although these species are readily distinguished even with only vegetative material. Ferdinand Mueller (one of the authors of Embothrium wickhamii), for example, determined a specimen of A. flammeum, collected in 1881 (near Trinity Bay, Karsten s.n.) as 'E. wickhami'. This confusion persisted until very recently (see e.g. Weston \& Crisp 1987: 22).

Selected sPecimens (36 examined): QueEnsland: Cook: Danbulla Forest Reserve Python Hill, $17^{\circ} 10^{\prime} \mathrm{S}, 145^{\circ} 40^{\prime} \mathrm{E}$, Stocker 944, 30 Aug 1972 (BRI, QRS); N of Malanda - Lake Barrine road,

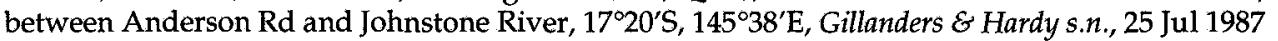

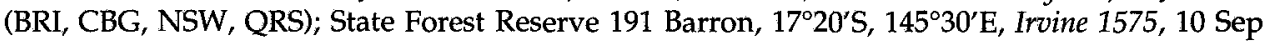
1975 (BRI, CANB, NSW, QRS); State Forest Reserve 194, East Barron Experimental Plot 38, $17^{\circ} 25^{\prime}$ S, $145^{\circ} 29^{\prime} \mathrm{E}$, Risley 219, 10 Jan 1978 (QRS). Cultivated: Bellingen (New South Wales), Crisp 7589 \& Taylor, 3 Nov 1984 (CBG, NSW).

\section{Alloxylon brachycarpum (Sleumer) P. Weston \& Crisp, comb. nov.}

BASIONYM: Embothrium brachycarpum Sleumer, Bot. Jahrb. Syst. 70: 130 (1939).

TYPE CITATION: 'Brit.-Papua: Lower Fly River, east bank opp. Sturt Island, ... Oct. 1936 (L.J. Brass n. 8170, Typus in Herb. Arnold Arboretum und Herb. Berlin).'

Lectotype (here designated): PAPUA NEw Guinea: Lower Fly River, east bank opp. Sturt Island, L.J. Brass 8170, Oct 1936 (B).

ISOLECTOTYPE: A (n.v.).

SYNONYM: Oreocallis brachycarpa (Sleumer) Sleumer, Bot. Jahrb. Syst. 76: 203 (1954); Sleumer, Fl. Males. ser. 1, 5: 201-203 (1955).

Illustration: Sleumer, Fl. Males. ser. 1, 5: Figure 24 (1955).

Seedlings and juveniles: seedling leaves simple, entire (D. Foreman pers. comm.); juveniles not known.

Adults: trees to $30 \mathrm{~m}$ high and $0.85 \mathrm{~m}$ d.b.h., sometimes slightly buttressed. Bark grey to brown, reticulately and shallowly fissured. Hairs minute, to $0.1 \mathrm{~mm}$, ferruginous, mostly appressed, straight. Young branchlets sparsely to moderately hairy, soon glabrescent. Leaves simple, entire, 5-16 cm long, 25-50 mm wide, discolorous, chartaceous; petiole 15-30 mm long, sparsely hairy when immature, glabrescent when mature; lamina elliptical to obovate to narrow-elliptical to narrow-obovate, forming angle of 45-75 degrees at base, rounded to obtuse, with flat to slightly recurved margins, sparsely hairy when very immature, soon glabrescent; venation brochidodromous, with secondary veins diverging at 40-70 degrees; midvein protruding slightly on adaxial surface and prominently on abaxial surface; lower order veins sunken. Conflorescences (2-)10-50-flowered, mostly axillary or ramiflorous, basitonic, mostly aggregated in superconflorescences of $2-8$ at ends of branches, strongly asymmetrical, pedicels curved so that all flowers are similarly oriented on same side of conflorescence axis; axis moderately to densely hairy; bracts of flower-pairs triangular to narrow-triangular to -oblong, usually caducous. Flowers pinkish red to bright red; pedicels 20-35 
$\mathrm{mm}$ long, moderately hairy; perianth $40-50 \mathrm{~mm}$ long, broadest below middle and tapering slightly to ends of claw, sparsely to moderately hairy on outside; pollen yellow; style c. as long as gynophore. Body of follicle 8-14 cm long; seeds 8-14.

FLOWERING PERIOD: June to October.

Distribution AND habITAT: Southern New Guinea; Upper Merauke River, Lower Fly River, Oriomo River and Aru Islands (Figure 3). At low altitudes on inland ridges or high banks of rivers not subject to inundation, usually in gallery rainforest but also recorded from bamboo-eucalypt forest (Sleumer 1955).

CONSERVATION STATUS: Not known; it is widespread in a relatively remote and undeveloped area of New Guinea.

Selected specimens (15 examined): Papua New Gunnea: Western District: Bensbach Sub-district,

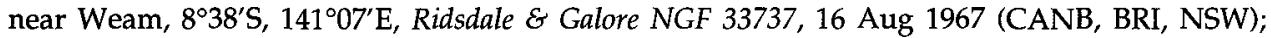

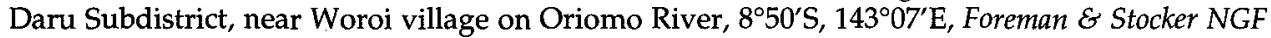
60384, 15 Jul 1974 (BRI, CANB, NSW, QRS); Morehead Subdistrict, $1 / 4$ mile [0.5 km] E of Karaita Village, $9^{\circ} 00^{\prime} \mathrm{S}, 141^{\circ} 25^{\prime} \mathrm{E}$, Eddowes \& Kumul NGF 36046, 17 Aug 1968 (BRI, CANB, NSW). INDONESIA: Irian Jaya: Bade, Bouman BW 3223, - (CANB).

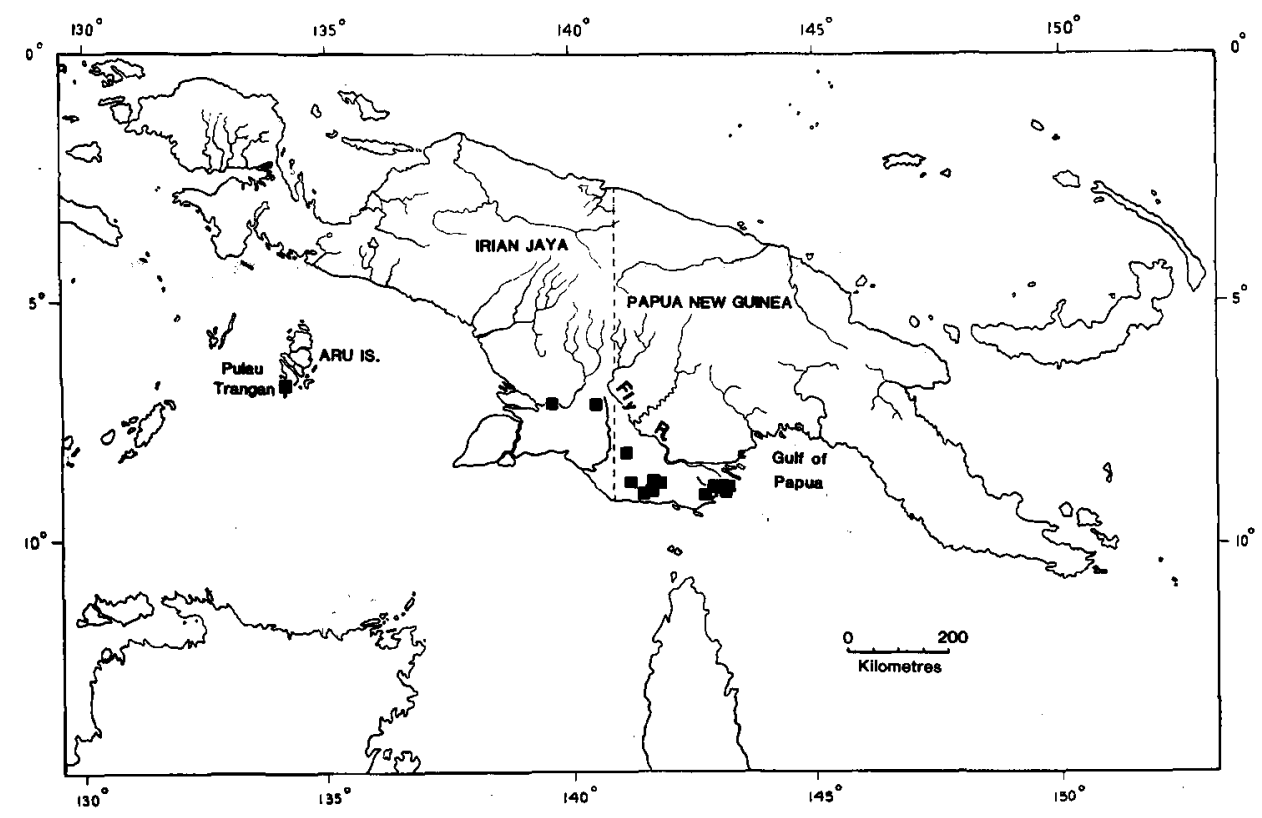

Figure 3. Distribution of A. brachycarpum. 


\section{Acknowledgments}

We are grateful to the following for assistance: the directors of B, BRI, CANB, MEL, $\mathrm{MO}$, and QRS for the loan of, or access to, their specimens of Embothriinae; Lawrie Johnson, Karen Wilson, Peter Wilson and an anonymous referee for critically reading the manuscript; David Mackay for drawing Figure 1 and Peter Richards and Dianne Godden for drawing Figures 2 and 3; the Australian Biological Resources Study for permitting us to publish figure 1; Garry Sankowsky for his assistance in the field in North Queensland and for supplying seedlings of A. flammeum and A. wickhamii; Penny Kater and the staff of the nursery, Royal Botanic Gardens Sydney, for maintaining our living collection of Alloxylon; Don Foreman for providing photos of flowering plants and data on seedlings of $A$. brachycarpum; Helen Hewson for helping with typification of $A$. wickhamii.

\section{References}

Chattaway, M.M. (1948) The wood anatomy of the Proteaceae. Austral. J. Sci. Res. (B) 1: 279-302. Hickey, L.J. (1973) Classification of the architecture of dicotyledonous leaves. Amer. J. Bot. 60: $17-33$.

Johnson, L.A.S. \& Briggs, B.G. (1963) Evolution in the Proteaceae. Austral. J. Bot. 11: 21-61.

Johnson, L.A.S. \& Briggs, B.G. (1975) On the Proteaceae - the evolution and classification of a southern family. Bot. J. Linn. Soc. 70: 83-182.

Sankowsky, G., Sankowsky, N., Radke, P. \& Radke, A. (1986) A field guide to the rainforest Proteaceae of north Queensland, edn 1 (The authors: Tolga)

Stearn, W.T. (1973) Botanical Latin, edn 2 (David \& Charles: Newton Abbot)

Sleumer, H. (1954) Proteaceae Americanae. Bot. Jahrb. Syst. 76: 139-211.

Sleumer, H. (1955) Proteaceae. Flora Malesiana ser. 1, 5: 147-206.

Weston, P.H. \& Crisp, M.D. (1987) Evolution and biogeography of the Waratahs. In J.A. Armstrong (ed.), Waratahs: their biology, cultivation and conservation. Australian National Botanic Gardens Occasional Publication No. 9. 